\title{
La práctica intelectual de los comisarios de la ciudad Buenos Aires (1812-1825)
}

The intellectual practice of the city commissioners Buenos Aires (1812-1825)

\author{
María Agustina Vaccaroni \\ Doctoranda - Centro de Estudios Historicos, Universidad Nacional de Mar del Plata / CONICET \\ magustinavaccaroni@gmail.com
}

\begin{abstract}
Resumen: En el presente artículo nos abocamos al examen de los comisarios de policía de la ciudad de Buenos Aires, realizando un ejercicio analítico sobre sus prácticas, cuestión que nos lleva a relacionarlas con "lo intelectual". Esto en tanto tenemos en cuenta su papel en la organización del control y la vigilancia, que los convierte en productores de institucionalidad, en un contexto de construcción conflictiva y contradictoria de las configuraciones policiales. Para dar cuenta de esto, nos valemos de fuentes diversas a las cuales recurrimos con un criterio de selección que focaliza sobre los actores involucrados. El período de referencia inicia con la instalación de los comisarios en 1812 como parte de la Intendencia de policía y acompaña su derrotero hasta 1825, cuando, con el Departamento de Policía ya establecido, se configuran las comisarías de ciudad.
\end{abstract}

Palabras clave: Comisario, práctica intelectual, configuraciones policiales.

\begin{abstract}
In this article we focus on the examination of the Buenos Aires city police deputies, by developing an analytical exercise on their practices, a question that leads us to relate them to intellectual aspects. This, in as much as we take into account their role in the organization of control and surveillance, which turns them into producers of institutionality, in a context of conflicting and contradictory construction of police configurations. To account for this, we use various sources, which we resort to through a selection criterion that focuses on the actors involved. The reference period begins with the installation of the police stations in 1812 as part of the Police Intendancy and accompanies their course until 1825, when, with the Police Department already established, the city police stations are configured.
\end{abstract}

Keywords: Deputy, intellectual practice, police configurations. 


\section{Introducción ${ }^{1}$}

Los comisarios de la ciudad de Buenos Aires fueron instalados en número de tres a partir de diciembre de 1812, junto con la sanción del Reglamento Provisional de Policía $^{2}$, el primero del tipo en la urbe porteña. Este último estableció una Intendencia de Policía, con un superior -el intendente de policía- dedicado a organizar este ramo, a quien los comisarios respondían. A su vez, una serie de "empleos"3 -tal la palabra que aparece en las fuentes- fueron creados para guarnecer de agentes a esta Intendencia, desde porteros hasta oficiales y una partida celadora de cien hombres ${ }^{4}$. Luego de 1821, con la transformación de la Intendencia en Departamento de Policía (REGISTRO OFICIAL, 1879: 125), los comisarios serán cuatro y eventualmente, pasarán a organizase como cabeza de las también cuatro comisarías que se instalan a lo largo del primer quinquenio de la década de 1820 en el área urbana.

La voz comisario ya se había usado en Buenos Aires en las primeras décadas del siglo XVIII para designar a los que luego serían los alcaldes de barrio desde 1772 (TAU ANZOÁTEGUI, 2004: 280-283) o para hablar de los agentes que eran parte del Tribunal del Santo Oficio, que poseía comisarios dedicados a una actividad vigilante cuya importancia "radicaba en su capacidad de generar denuncias" (MIRANDA OJEDA, 2013: 11). Diferentes de aquellos, los comisarios aquí referidos accionan en base a la delegación de autoridad realizada por parte del intendente, a quien deben responder y cuyas órdenes deben efectivizar. Esto bien coincide con la definición del período sobre la voz comisario que lo señala como "el que tiene poder y facultad de otro

\footnotetext{
${ }^{1}$ El artículo presentado retoma algunas preocupaciones analizadas en el marco de nuestra tesis doctoral, pronta a ser presentada para su defensa. La misma, como el presente artículo, es financiada a través de una beca de finalización de Doctorado otorgada por el Consejo Nacional de Investigaciones Científicas y técnicas de Argentina (CONICET).

${ }^{2}$ El mismo se encuentra transcripto en Prado y Rojas (1877: 150-157). Su redacción corresponde a hombres asociados a la Sociedad Patriótica como José Moldes e Hipólito Vieytes, y se produce a iniciativa del Segundo Triunvirato que arriba al poder luego del movimiento de pueblo del 8 de octubre de 1812 (HERRERO, 2007).

${ }^{3}$ En el Diccionario de la Real Academia de 1817 (también aparece la misma definición en 1803 y 1822) la voz empleo es referida como puesto, oficio y ocupación. A su vez, se amplía el concepto señalando que emplear es ocupar a uno encargándole algún negocio o comisión. (REAL ACADEMIA, 1817: 216).

${ }^{4}$ Documentos firmados por los comisarios dan cuenta de que la cantidad de miembros de la partida celadora efectivamente oscilaba entre ochenta y nueve y noventa y dos hombres (AGN, 1831-1834-1815). Aclaramos que en lo que sigue y a los fines de una mejor comprensión, hemos modernizado la grafía cada vez que transcribimos parte de las fuentes consultadas.
} 
para ejecutar alguna orden, o entender alguna negocio", relacionado así con la palabra delegatus (REAL ACADEMIA, 1780: 246).

A pesar de ser un engranaje importante de las configuraciones policiales ${ }^{5}$ en construcción durante la década revolucionaria y el proyecto rivadaviano ${ }^{6}$, el abordaje durante el período sobre los comisarios es escaso. Para articular nuestro objeto de estudio aquí nos hemos valido de autores cuyos trabajos retoman las relaciones conflictivas entre justicia y policía (BARRENECHE, 2001) y entre derecho y policía (CASAGRANDE, 2019). Desde la historia social de la justicia y el gobierno significativos aportes han sido desenvueltos al respecto, los cuales, además, se retroalimentaron de lo hecho al otro lado del Atlántico, por caso, en estudios como el considerado pionero de Steven Kaplan (1981). Su señalamiento sobre los comisarios como agentes de mediación y contacto cotidiano con la población se condice con el uso que ha hecho Arlette Farge (2008) de sus memorias, a los fines de indagar sobre el día a día del pueblo parisino en la Francia revolucionaria.

A su vez, diversos estudios locales para décadas posteriores a las tratadas en el presente trabajo, tanto en el ámbito urbano como rural, han constituido un importante insumo para afinar la perspectiva de nuestro análisis. Para la ciudad, los estudios realizados comprenden mayormente los decenios correspondientes a la segunda parte del siglo XIX, período para el cual se han analizado la composición del plantel policial con sus características de constante deserción, analfabetismo e indisciplinas (GAYOL, 1996). También las reformas del último tercio decimonónico han sido atendidas, en especial a partir de la Ley de Municipalidades en 1854, para desentrañar cómo una policía considerada monstruosa por sus contemporáneos fue experimentado procesos de reorganización (GALEANO, 2015: 193). Un poco más adelante, estudios como el de Berardi (2018) se ocuparon de la creación de la policía de la provincia de Buenos Aires hacia 1880 y sus relaciones con la política.

\footnotetext{
${ }^{5}$ Optamos por la noción de configuraciones en tanto hablamos de diversos elementos que, en su disposición y forma, hacen a un todo que está en proceso de construcción, que muestra ciertas particularidades pero que lejos está, en esta etapa, de considerarse una arquitectura institucional profesionalizada.

${ }^{6}$ Remitimos al período que se conoce ampliamente como la "feliz experiencia", que abarca la primera parte de la década de 1820 cuando se ponen en marcha una serie de reformas, de inspiración liberal y contenido modernizador, tanto en el aspecto militar, como en el eclesiástico, económico y político (TERNAVASIO, 2004). Autores como Fabián Herrero han señalado, sin embargo, que el reformismo rivadaviano se vio envuelto en un clima de tensiones y conflictos. En sus palabras, "la oleada reformista que afecta a autoridades (Cabildo), territorios (expansión frontera y política con los indios), sujetos e instituciones (reformas del clero, militar, imprenta) no se dio en un clima de relativa paz y de ausencia de facciones, por el contrario, ocurrió en una escena abierta y dinámica atravesada y rodeada por distintos movimientos de redes, de grupos y fracciones políticas" (2018: 120).
} 
Quienes han tenido en cuenta la acción de los comisarios en la campaña han retrocedido hasta la década de 1830 en sus análisis, poniendo de manifiesto la relación con el territorio (FRADKIN, 2008; IRAOLAGOITIA, 2014; YANGILEVICH, 2018), y los conflictos y la superposición y acumulación de funciones con otros agentes, como los jueces de paz (RICO, 2007), así como sus perfiles y trayectorias (PIAZZI Y RÍOS, 2012).

Para la década y media que ataña a este estudio, no podemos dejar de mencionar el aporte que han significado los estudios abocados al análisis de las justicias menores y de proximidad en diferentes espacios, como los alcaldes de hermandad (LEVAGGI, 2009; BARRIERA, 2013), los alcaldes de barrio y decuriones (DÍAZ DE ZAPPIA, 2008; BARRIERA, 2017, MOLINA, 2010) y, de nuevo, la justicia de paz (GASTELLU, 2018), entre otros. Estas y otras indagaciones han retomado el señalamiento de Barriera sobre la necesidad de abordar, para estas problemáticas, un examen exhaustivo del gobierno, la justicia y la policía y sobre "quiénes fueron sus agentes, qué pensaban y cómo se comportaban pero también saber con quiénes y cómo estaban relacionados, cómo convivían con otras autoridades, con sus convecinos y, desde luego, cómo eran percibidos por todos ellos" (2010: 58).

Parte de esta tarea es la que retomamos aquí al respecto de estos comisarios de ciudad que, hemos visto, aun no son tan conocidos. A través de un análisis sobre sus tareas cotidianas y sus relaciones con el intendente de policía, con el gobierno, con otros agentes con funciones policiales y con los habitantes porteños tratamos de conceptualizar su actividad en relación a "lo intelectual". Entendemos esto último como un aspecto relacional y que no está circunscripto a una capa específica de personas con cierta educación, renombre y reputación. En efecto, lo intelectual forma parte de la acción cotidiana de los comisarios analizados en tanto ejercen "funciones organizativas en sentido lato, tanto en el campo de la producción como en el de la cultura y en el político-administrativo" (GRAMSCI, 1999: 412). Los comisarios de ciudad no se limitan a ejecutar las órdenes del intendente de policía y el gobierno, aun cuando este así lo demande, sino que participan en la confección de las disposiciones relativas a la policía, sea proponiendo el articulado de determinados reglamentos, realizando informes y sugerencias sobre la actuación de otros agentes, y siendo comisionados para organizar pueblos enteros.

Si bien el uso del término "intelectual" se puede rastrear hacia el último tercio del siglo XIX, y muchos autores ubican su piedra fundacional a partir del caso Dreyfus 
en la Francia de 1898, cuando se publica el manifiesto de Emile Zola y el petitorio de los clercs (PROCHASSON, 2003; ALTAMIRANO, 2013), diversos autores dedicados a la historia hispano y luso americana han retomado su uso en tanto categoría analítica. Por caso, Marieta Pinheiro de Carvalho (2007) se ha ocupado de reconstruir las relaciones establecidas entre el Estado portugués y los intelectuales lusobrasileros hacia fines del siglo XVIII, analizándolas en conexión con el traslado de la Corte portuguesa a Río de Janeiro luego de los eventos de 1808. Para ella, la crisis originada con la invasión napoleónica a la península acentúa la participación de estos intelectuales en los temas de Estado ${ }^{7}$. Por su parte, y centrado en Buenos Aires, Mariano Schlez (2013: 872) también ha hecho uso de este concepto para explicar de qué modo el gran comerciante Diego de Agüero “desempeñó funciones tanto, desde un lugar de dirección, planteó un balance y una salida, es decir, un programa: la defensa irrestricta de la monarquía española y el status quo colonial". Para este autor, lo intelectual estuvo directamente asociado a la situación material de los actores involucrados (SCHLEZ, 2010: 123).

Retomamos estos planteamientos para indagar sobre el modo en que los comisarios que estudiamos realizan funciones organizativas del ramo de policía asociadas a "lo intelectual", en tanto son estos comisarios quienes, en gran parte, "producen" la institucionalidad vinculada al control y la vigilancia. Para dar cuenta de esta tesis utilizamos diversas fuentes y documentos que entrecruzamos y que incluyen órdenes superiores ${ }^{8}$-partes diarios e informativos de la policía contenidos en el Fondo de Policía ubicado en la Sala X del Archivo General de la Nación-, fondos de lotería y multas, juicios criminales -provenientes del Fondo de Juzgado del Crimen del Archivo Histórico de la provincia de Buenos Aires-, acuerdos del Cabildo, reglamentaciones y legislación del período. El criterio de selección reside en la focalización de la problemática y los actores en cuestión.

De este modo, en los siguientes párrafos explicamos cómo se instalan estos comisarios en la ciudad a partir de 1812 y sus diferencias con la superioridad policial y con otros agentes subalternos, cuestión que los ubica "en el medio" de las configuraciones policiales del momento. Pasamos luego a profundizar sobre sus relaciones con el proceso revolucionario y la reactualización de sus trayectorias durante

\footnotetext{
${ }^{7}$ Pimenta (2013), aunque ocupado en discutir el derrotero brasilero en comparación con el rioplatense en el marco de las discusiones por las independencias y las guerras que le siguen, da cuenta del papel que jugaron las noticias, rumores y pronósticos en las propuestas juntistas en Brasil, que fueron seguidas muy de cerca por la alta política imperial portuguesa en donde actuaban estos intelectuales ilustrados que conciliaron practicas iluministas e ideas absolutistas.

${ }^{8}$ Un análisis específico sobre ellas en Vaccaroni (2014).
} 
el primer lustro de la década de 1820. Finalmente, damos cuenta de las tareas que realizaban, retomando su propia voz en los documentos, para analizar el modo en que se construyen como productores de institucionalidad. Realizamos un corte en 1825, en primer lugar, porque condensa una serie de eventos políticos de importancia política (TERNAVASIO, 2004: 40$)^{9}$ pero también cambios significativos en términos institucionales para la propia policía. Esto en tanto en febrero de ese año se suprimen las comisarías de campaña, para luego ser reinstaladas poco tiempo después, en junio, pero ahora con un reglamento propio, dando indicios de una primera diferenciación entre el poder de policía que emanaba desde el casco urbano y una policía particular para el ámbito rural ${ }^{10}$. En este mismo momento es que se consolidan las comisarías de ciudad como el elemento medular de la actividad policial en el espacio urbano, no sólo con la conformación del reglamento (MANUAL, 1830: 96-98) que regula su funcionamiento sino también con la instalación de casas de policía, alquiladas y diferentes del hogar particular de los agentes policiales y con su propio mobiliario e insumos ${ }^{11}$.

\section{Agentes en el medio}

Entre las nuevas formas de mirar a la policía se encuentra el análisis sobre sus agentes, sus trayectorias, perfiles y posiciones socioeconómicas (PIAZZI Y RÍOS, 2012: 381), a los fines de acercarse a los lugares y posiciones ocupadas en distintos espacios institucionales. En la Argentina, este tipo de estudios habían sido, generalmente, más abundantes entre los historiadores políticos y más recientemente entre los historiadores sociales dedicados a la justicia (LANTERI, 2015; CANDIOTI, 2017). No obstante, en los últimos años y como ya ha sucedido en Europa, cada vez más

\footnotetext{
${ }^{9}$ Para este momento ha sido reemplazado el gobernador Martín Rodríguez por Juan Gregorio de Las Heras, y por ende se pone fin a la actuación ministerial de Rivadavia. En simultáneo, se desarrolla el Congreso Constituyente de 1824-1826 que, como señala Ternavasio marca -como no había sucedido antes- una divisoria dentro de las elites gobernantes.

${ }^{10}$ Estos cambios sobrevienen alrededor de los años en que Candioti identifica, en referencia a la administración de justicia, la existencia de "una conciencia más clara de que la tarea de juzgar era una función específica que, si bien podía coincidir o ser ejercida simultáneamente a tareas de gobierno o de representación de los conciudadanos, ya no se identificaba con ellas ni tenía la misma fuente de legitimidad. De esta forma, un aspecto central de la plurisecular cultura jurisdiccional se había roto definitivamente" (2017: 232). Al mismo tiempo, situando su mirada en la campaña, Fradkin señala que allí impacta un doble fracaso durante la década de 1820 en torno a la iniciativa de organizar "sistemas de justicia y policía diferenciados" (2008: 249)

${ }^{11}$ Por estos años que la jurisdicción citadina se amplía y se reconfiguran los cuarteles, agregando diez más a los ya treinta y tres existentes, que mantienen una vinculación fluida entre las áreas urbanas, periurbanas y rurales que en el momento se encontraban formando parte de un mismo complejo productivo, mercantil, institucional y político (CILIBERTO Y ROSAS PRINCIPI, 2014: 281).
} 
análisis historiográficos se interesan por saber quiénes eran los agentes policiales actuantes. Carolina Piazzi y Evangelina Ríos (2018: 402) se han interesado por los perfiles de los comisarios de la campaña santafesina, a quienes describen como "personas influyentes en el ámbito local que tenían experiencia en el manejo de asuntos tanto políticos como económicos, y que contaban con la confianza del resto de los hacendados". Para el espacio rioplatense, la mirada de los comisarios ha sido valorada como parte del dispositivo de administración del espacio urbano de la segunda mitad del XIX, mostrando un enfoque sobre la policía que la concibe como un conglomerado de actores con trayectorias singulares y con agencia histórica (GALEANO, 2017a: 17). Para áreas cercanas, contamos con los estudios de Nicolás Duffau (2018) que ha señalado la duplicidad de funciones de los comisarios orientales que oscilaban entre la vigilancia y el mantenimiento del orden urbano, mientras que Marcos Bretas, para el espacio luso, ha analizado la policía de Río de Janeiro, dando lugar a una tesis que comprende a los agentes policiales como elementos insertos en el sistema de dominación pero también como víctimas del reclutamiento forzado y participantes de los “dramas cotidianos" (2011: 90).

Estos desarrollos analíticos parecen tener en común la idea del comisario como "homme entredeux", tal como lo definen para la Francia del siglo XVIII y XIX Dominique Kalifa y Pierre Karina Cohen (2008: 17). Para dar cuenta de los comisarios como estos "hombres en el medio" en la ciudad de Buenos Aires para los años señalados en este trabajo, reconstruimos tres variables asociadas a las dotaciones que reciben por su tarea, a su papel en los procesos judiciales y a su establecimiento como cabeza de las comisarías que se configuran en la primera mitad de 1820.

Los comisarios a los que atendemos cumplen tareas de policía de acuerdo al Reglamento de 1812 que hemos mencionado, el cual expresa una noción de policía que está en transformación en el período, pero que aún remite al sentido antiguo del buen gobierno de ciudades y repúblicas. La voz "policía" designa un concepto amplio, correspondiente con el buen orden de la ciudad, y se desarrolla en el período "bajo la forma de reglamentos, obras y creación de jurisdicciones, $[\ldots]$ la policía de fomento y promoción económica, la policía de la moral, policía vial y edilicia, de abastos" (FAVELUKES, 2007: 8). Sus tareas, así planteadas, corresponden al objetivo de conservar la ciudad y a su población en quietud y sosiego, es decir, en armonía (CASAGRANDE, 2015: 50-51), con arreglo a la perspectiva del orden jurídico-político del período. En cierta forma (o en todas) es parte de una "manera de gobernar" 
(FOUCAULT, 2006: 357) y una "economía política urbana” por lo cual sus tareas pueden incluir "el mantenimiento de las vías de comunicación, el transporte de mercancías y todo lo indispensable para el aprovisionamiento de las ciudades" (ALZATE ECHEVERRI, 2007: 37). Por ello, la actividad de los comisarios combina una preocupación por el control de los sectores populares y los opositores políticos, con funciones de organización, ordenamiento e higiene urbana. Las funciones delimitadas para estos comisarios integran así la potestad doméstica, civil y gubernativa en toda la extensión de la capital ${ }^{12}$, ocupándose entre otras cosas del trabajo de enlozado de la Recova, del celo sobre las obras de teatro producidas, de entablar relaciones con las comunidades de negros, entre otras cuestiones.

A diferencia de los alcaldes de barrio cuya función es considerada una carga pública, el del comisario es considerado uno de los empleos que, de acuerdo al reglamento policial, recibe una gratificación. Para el intendente de policía se establece en la normativa una dotación de $\$ 3.000$ anuales $^{13}$. Este número decrece según se trate de oficiales o comisarios. Los primeros tres comisarios nombrados en 1812, Miguel Antonio Sáenz, Francisco Doblas y Felipe Robles, aparecen en la documentación percibiendo \$ 100 mensuales, es decir, \$ 1.200 anuales (AGN, 1812-1822), asentando sus firmas de "recibido" en un registro (AGN, 1813). Aún con una dotación reducida a partir de 1822, cuando se amplía el número de comisarios a cuatro -los nombrados en este caso son de nuevo Miguel Antonio Sáenz acompañado de Prudencio Sagari, Modesto Sánchez y Agustín Herrera- y se reduce su dotación a \$800 cada uno (REGISTRO OFICIAL, 1879: 125), los comisarios siguen obteniendo dinero a cambio del cumplimiento de su función durante todo el período. Destaca, a su vez, que, en comparación con los alcaldes pero también con respecto a los intendentes / jefes, tiene una permanencia considerable en sus empleos. Comisarios como Doblas o Herrera se mantienen en su cargo por ocho años, Robles y Sagari por seis, mientras que Sáenz seguía siendo comisario tesorero en 1825, ostentando la titularidad de este puesto por casi quince años ${ }^{14}$. En suma, si bien son subalternos del intendente / jefe, su actividad

\footnotetext{
${ }^{12}$ Esta potestas, esto es, autoridad, se construirá en tensión con la perspectiva jurisdiccional de la justicia, en tanto la primera implicaba la acción de ejecutar y prevenir, no de evaluar, interpretar y punir (CASAGRANDE, 2019: 22).

${ }^{13}$ Todas las expresiones monetarias corresponden a pesos corrientes.

14 Los comisarios de campaña también parecen haber permanecido en sus empleos por períodos considerables. Incluso la mayoría de los comisarios destituidos en 1825 y que habían sido designados entre 1822 y 1824 son los mismos que luego son repuestos en sus cargos más adelante en el mismo año de 1825 (LEVAGGI, 1976: 403-404).
} 
no es considerada una carga honorífica en tanto vecinos, sino una ocupación reglamentada y remunerada.

Policía y justicia en este período aparecen muchas veces sobreimpresas e intercaladas. Ciertamente, los agentes con funciones policiales son quienes dan inicio a los procesos judiciales, al redactar los sumarios o partes que describen el hecho criminal a ser juzgado. Quienes están encargados de esto son, primero, los alcaldes de barrio, quienes también son muchas veces enviados a arrestar a sospechosos (AHPBA, 1812) o hacer averiguaciones diversas sobre lo ocurrido en robos, pleitos y homicidios (AHPBA, 1813). Sin embargo, son los comisarios quienes reciben estos primeros informes y los envían al intendente o jefe de policía. Así lo expresa el comisario Modesto Sánchez que informa al jefe de policía sobre un robo, señalando que no ha “ocurrido en la sección que está a su cargo más novedad que la prisión hecha por el Alcalde del Cuartel 28 a Pedro Ramírez por haberle robado a Juan Manuel Ruiz cuatro caballos, dos yeguas y la marca" (AHPBA, 1823). Claro que muchas veces esto genera conflictos. Según Sofía Gastellu (2019: 101), en 1830 esta facultad para levantar y elevar sumarios es revocada por decreto del gobierno, pero su efecto real se constata mucho más lento, manteniendo a los comisarios en esta posición de mediadores entre los agentes policiales que caminan el terreno, como los alcaldes de barrio, y la superioridad policial, la administración de justicia y el gobierno.

Esta posición se reactualiza y refuerza desde 1822, con el proyecto de creación de las comisarías de sección, que implica la división de la ciudad en cuatro secciones o departamentos (AGN, 1822). Si bien el alquiler de espacios específicos, las casas de policía, donde eventualmente se nuclean diferentes agentes policiales de la sección se verifica hacia 1826, año en que el gobierno ordena construir cuatro mesas para "los comisarios de sección” (ÍNDICE, 1959: 158), la organización de las comisarías se extiende durante todo el primer lustro de la década de 1820 . Por ello entre 1822 y 1824 se nombran nuevos empleos y agentes que se articulan bajo el mando de los comisarios. Así sucede con los comisarios segundos, que deben escoltar a los comisarios a la cabeza de cada sección y con los médicos, nombrados en número de cuatro y cuyo servicio debía ser anual y gratuito (REGISTRO OFICIAL, 1879: 75-76). Además, desde 1824, cada sección o departamento cuenta con ocho celadores. El reglamento que los arregla establece que "los comisarios de las secciones respectivas [que, N.A] están especialmente encargados de cuidar que los celadores empleen las horas del día en solo el servicio público" (REGISTRO OFCIAL, 1879: 6). Todos los nuevos nombramientos, 
algunos acompañados con dotaciones y otros sin ellas, quedarían bajo la supervisión y las órdenes del jefe del Departamento de Policía, pero a la vez, expresamente indicados para secundar la actividad en las comisarías.

La posición intermedia de los comisarios, como canal de comunicación entre agentes subalternos y la superioridad policial y como organizadores de diversos auxiliares en las comisarías puede haber sido un insumo que alienta tareas de tipo intelectual en tanto organizacionales de las configuraciones policiales en construcción. La mediación comisarial también debió haber sido impulsada y a la vez impactada por los saberes, cocimientos y redes que poseen los comisarios al ingresar a la policía.

\section{De la Revolución a Rivadavia}

Los comisarios, además de ser un núcleo organizador de la práctica cotidiana de diferentes agentes con funciones policiales, también estaban insertos en diferentes redes políticas y familiares que se habían construido en los entresijos del gobierno colonial, de la defensa de Buenos Aires en 1806 y 1807 y del proceso revolucionario abierto en 1810. Luego de la supresión del Cabildo en 1821, nuevos comisarios supieron utilizar los saberes adquiridos en aquellos procesos para ponerlos al servicio de una policía que pretendía ser reformada ${ }^{15}$.

Quienes actúan durante la década revolucionaria provienen de familias de comerciantes importantes y que también han formado parte del gobierno. En el caso del comisario Francisco Doblas, su padre Gonzalo, emigrante andaluz, figura formando parte de una compañía con Gaspar Santa Coloma (GARCÍA DE FLOEL, 2000: 45), reconocido comerciante de efectos de Castilla y asociado al clan Basavilbaso. Posteriormente, es teniente gobernador del territorio de Misiones, sobre el cual escribe una Memoria histórica enviada al ingeniero, arquitecto y militar Félix de Azara, y que luego llegó a ser presentada -aunque finalmente rechazada- en la corte de Carlos III. Por su parte, la familia del comisario Miguel Antonio Sáenz, comerciantes de Castilla La Vieja, incluía a su hermano mayor, el presbítero Antonio, secretario del Cabildo Eclesiástico, primer rector de la Universidad de Buenos Aires y miembro de la Sociedad

\footnotetext{
${ }^{15}$ Los datos biográficos y asociados a las trayectorias y perfiles de los comisarios fueron reconstruidos en base a información proveniente de extraídos de los Acuerdos de Extinguido Cabildo de Buenos Aires, del Registro Oficial de la Provincia de Buenos Aires, de AGN, 1812-1822, AGN, 1822, AGN, 1823, AGN, 1824, AGN, 1825-1826, y de diccionarios biográficos compilados por autores como Cutolo (1968), Yaben (1938), Muzzio (1920) y Piccirili, Romay y Gianello (1953).
} 
Patriótica. Mientras tanto el comisario Felipe Robles, de quien no tenemos datos sobre su familia, sí había sido el mismo alcalde de barrio del cuartel 10 en 1811 y administrador de la obra del nuevo Coliseo, empleo al que renuncia para convertirse en comisario (ACUERDOS, 1813-1814: 207). Previamente, habría logrado una serie de conocimientos sobre las manzanas de la ciudad como asentista de la obra de alumbrado, cuyo remate ganó entre los años 1791 a 1797, con la aprobación del Cabildo. Esta tarea, además de una retribución económica, puede haberle ganado a Robles una serie de saberes sobre los pobladores que sirvieron para su actividad comisarial en tanto los asentistas recurrían a un pequeño grupo de personas para realizar sus tareas y luego debían ellos mismos "llevar a cabo el cobro del coste", vecino por vecino ${ }^{16}$.

Otros, como Prudencio Sagari ocupado como comisario desde 1822, también poseen experiencias interesantes que pueden haber sido capitalizadas en este rol y que han sido desarrolladas al calor de los eventos de la década revolucionaria. Sagari, por caso, había sido alcalde de hermandad de la Banda del Sur en 1817 -reelecto en 1818 administrador de la Imprenta del Estado, al menos hasta 1826, en forma paralela a su papel como comisario. También había cumplido tareas como mayordomo de la obra de enlozado de la Recova un tiempo antes, y desde 1826 es nombrado ecónomo del hospital de mujeres con un "sueldo de mil pesos anuales" (REGISTRO NACIONAL, 1826: 238). El caso de Agustín Herrera, también nombrado en 1822, se asocia con la dimensión militar, en tanto había estudiado en la Escuela de Náutica del Consulado de Buenos Aires, parar luego ingresar al Regimiento Fijo de Buenos Aires, donde participó en la defensa de la ciudad durante la segunda invasión inglesa. Esta dimensión es compartida con el comisario Modesto Sánchez, que forma parte del $3^{\circ}$ Escuadrón de Húsares, en donde reviste como cadete en 1807. Tanto Herrera como Sánchez terminarán alcanzando el grado de Capitán, de Artillería y del Regimiento nº respectivamente. Sáenz y Doblas compartían su paso por el servicio miliciano: ambos habían sido miembros del Escuadrón de Húsares y, como sus pares, habían participado repeliendo las invasiones inglesas.

Así como participan de la defensa de Buenos Aires en 1806-1807, también varios de los comisarios analizados tienen intervención en los eventos revolucionarios. Sáenz participa del Cabildo abierto del 22 de mayo de 1810, votando a favor para que

\footnotetext{
${ }^{16}$ Los asentistas de obras públicas se correspondían con una forma de gestión supervisada por el Cabildo o Ayuntamiento pero en las manos concretas de "privados" que accedían a estos trabajos por medio de subasta pública (MADRID, 2007: 28).
} 
cese el virrey (ACUERDOS, 1810-1811: 118). Junto con Robles, que había sido designado alcalde de barrio luego de la remoción de las anteriores autoridades, hecha por la Junta Gubernativa (CANDIOTI, 2017: 50), firman la proclama contra el Primer Triunvirato (JUNTA, 1812: 313). Estas lealtades públicas son acompañadas, en ocasiones, por compromisos monetarios: por ello, Sáenz y Doblas donan una onza cada uno "por la causa de la patria" para pagar armamento (JUNTA, 1812: 214). Todos ellos, sumados a Sagari, que había peleado en el Ejército del Norte, son considerados vecinos americanos, en tanto son convocados a votar en septiembre de 1811 en ocasión de la elección de asesores del gobierno (ACUERDOS, 1810-1811: 559). En algunos casos, los comisarios son recompensados por su adhesión y servicio, por ejemplo Felipe Robles, quien es ubicado por María Elena Infesta (2006: 73) como uno de los receptores de donaciones de tierras públicas hechas por el Directorio.

La proveniencia de familias asociadas al comercio y al gobierno, su participación definitoria en la defensa de Buenos Aires y su adhesión y alineamiento político preciso es reflejo de su inserción en redes económicas, políticas y familiares, cuestión que, por supuesto, colabora en el desempeño de su rol como mediadores. En este sentido, Diego Galeano destaca que "además del anclaje estrictamente local y barrial del comisario, su figura involucra una dimensión global: objeto de circulaciones que cruzaban fronteras, de complejos procesos de traducción y de diálogos atravesados por miradas críticas" (2017b: 14). Estos procesos de transacción, traducción, intercambio y diálogo son posibles y se replican en su papel como productores de institucionalidad.

\section{Productores de institucionalidad}

Si bien no podemos considerar a los comisarios analizados como intelectuales lisa y llanamente, tampoco podemos dejar de reflexionar sobre parte de su actividad como una de tipo intelectual. Los comisarios practican lo intelectual en tanto productores de institucionalidad -no los únicos- de cierta importancia en el marco de las configuraciones policiales en construcción. Son, a la vez, delegados del intendente / jefe y conocen, poco a poco, a segmentos considerables de la población a la que vigilan, situación que permite la elevación de quejas, recomendaciones y sugerencias a la 
superioridad policial sobre cómo organizar el control al respecto de los diferentes grupos que consideran peligrosos.

Veamos los reglamentos, en tanto muchos de ellos se originan en la cotidiana comunicación que los comisarios mantienen con la superioridad policial y por su intermedio con el gobierno. Esta comunicación no es unidireccional, sino que implica una atención por parte de los intendentes y jefes de policía que las reciben. Tal es el caso del Reglamento de Lotería, redactado por Doblas en 1815. El comisario extiende al gobierno una evaluación sobre el ramo, en donde a lo largo de diez puntos señala cómo debe organizarse la lotería a los fines de, por un lado, aliviar la "decadencia" de los ingresos de la policía, y por el otro, quitar a los empresarios particulares su administración (AGN, 1812-1822). El reglamento mencionado en aprobado tal como lo elabora Doblas, es decir que, en este caso, es uno de los comisarios quien proyecta los modos en que se debe disponer el juego para evitar sus excesos y una mala administración. Las recomendaciones del comisario Sáenz también son escuchadas por el gobierno, en ocasión, por ejemplo, de las órdenes libradas para vigilar las costumbres y hábitos de las festividades y bailes realizados por la nación conga. En su informe, Miguel Antonio nombra y describe a sus líderes, las formas que asumían las festividades y los cobros que se realizaban en las mismas, otorgando datos que luego el jefe Achával utiliza para expedir un reglamento de ocho artículos sobre cómo debían proceder los bailes de samba en el cuartel 15. Los artículos cuarto y quinto son copia textual de propuestas hechas por Sáenz en su informe inicial (AGN, 1812-1822). La preocupación por estas comunidades, que se remonta a las invasiones inglesas y el peligro que se experimenta por parte de los capitulares y la dirigencia del momento al haber armado a "la esclavatura y plebe" (ACUERDOS, 1805-1807: 686), se traslada también a la década de 1820. En ese contexto cuando los procesos de reformulación de los derechos de la tierra (CILIBERTO, 2009: 138) chocan con la proliferación de los llamados vagos y malentretenidos, que más bien eran jornaleros y labradores (ALONSO, 2001), los comisarios vuelven a actuar. Por eso, en la década de 1820, una situación similar envuelve al comisario Modesto Sánchez que hace recomendaciones al nuevo jefe de policía Hipólito Videla sobre las reglamentaciones en torno a las sociedades negras (AGN, 1825-1826).

En estas cuestiones es posible ver un entrelazamiento entre la producción de institucionalidad, con objetivos específicos que están ligados a la dirigencia del momento. Las tareas señaladas equipan a los comisarios con experiencias y 
conocimientos que aparecen como un valor para los jefes y el propio gobierno, y que se adicionan a los saberes que los comisarios traen como parte de las redes política sy familaires que hemos mencionado. Asimismo, no pueden sino asociarse a la producción de institucionalidad, hecha "desde abajo". Muchas veces esto deriva en el nombramiento o destitución de otros agentes más o menos asociados a la policía. Esto sucede cuando el comisario Prudencio Sagari en 1821 eleva un informe a Rivadavia sobre Manuel Collans, que deriva en su desvinculación como asentista del alumbrado (SZUCHMAN, 2001: 204). O cuando el comisario Perichon hace la recomendación de un sujeto detemrinado para ocupar el cargo del alcalde (AGN, 1825-1826). El comisario Sáenz, por su parte, avanza y no sólo hace saber su opinión al intendente en 1813 sobre ciertos individuos sino sobre un espacio institucional entero al mostrar su fuerte desacuerdo con los gastos y la manera de conducirse de las presas y los auxiliares en la Casa de Corrigendas. Argumenta que:

no deben ser de abono a la Rectora de la Casa de Corrigendas las partidas de almidón, pago de peón para llevar las velas, y compra de encaje las que suman la cantidad de ocho reales por las razones siguientes: el almidón no deben gastarlo las presas porque es superfluo y por lo tanto no se les ha señalado, las velas deben conducirlas las ordenanzas pues están para cuanto ocurra que hacer en dicha casa; y el encaje no debe comprarlo de los fondos de la Casa por haber ordenado el Superior Poder Ejecutivo a los ministros generales Hacienda (AGN, 1813).

Al mismo tiempo, la acción de los comisarios comporta un mecanismo importante para que la jefatura conozca los reclamos, situaciones y cotidianeidad de los vecinos, cuestión que se evidencia cuando el jefe de Policía Joaquín de Achával evalúa una propuesta de edificación en tanto le "ha informado el Comisario de Policía Don Agustín Herrera es acequible y ventajosa" (AHPBA, 1822). Esto es posible en tanto los comisarios, en ocasiones, también recorren el terreno como los acaldes de barrio. Ejemplo de ello es la comisión hecha al comisario Felipe Robles cuando se apresta a acompañar y supervisar la demarcación del pueblo de los Quilmes hacia donde parte “asociado de don Francisco Mesura, dos ayudantes de este, y del Escribano Don Manuel José Godoy el 22 de febrero de 1818” (QUESADA, 1942: 70). 
Estas relaciones en las que los comisarios intervienen no sólo ejecutando sino también produciendo opiniones, sugerencias, informes, no están exentas de críticas y conflictos. Las propuestas de Doblas para una mejor circulación en los puestos de abastos ubicados en la Plaza de la Resistencia son consideradas por el Fiel Ejecutor del Cabildo, Fermín de Tocornal como un "ultraje y desaire con que ha tratado a su Juzgado el comisionado de la Policía Don Francisco Doblas", por haber puesto pie sobre su jurisdicción (ACUERDOS, 1813-1814: 660). El decreto del gobierno que inicia la instalación de las comisarías de ciudad, de hecho, es inaugurado con críticas a la actuación de los comisarios. Rivadavia hace hincapié en que su tarea es la de "hacer ejecutar", porque:

ya transcursado con período de tiempo suficiente para que los comisarios de Policía estén en la aptitud que demandan los objetos de su institución, y el lleno cumplimiento de sus deberes, el Gobierno quiere y espera que en adelante el servicio de los expresados comisarios no dé lugar a reconvenciones que la Superioridad desearía siempre evitar (AGN, 1822).

Aun con estos conflictos en desarrollo, es posible señalar que los comisarios realizan diversas tareas y entre ellas algunas que superan la ejecución de disposiciones. Muchas veces son parte de las creación de estas últimas, mediante la presentación e introducción a los intendentes y jefes de quejas, reclamos, sugerencias o directamente la confección del articulado de diversos reglamentos. Estos modos de pensar el control y la vigilancia hacen de los comisarios parte importante de la producción de una institucionalidad policial que se encuentra en plena construcción. Estas prácticas, de tipo intelectual, que desarrollan los comisarios analizados, están impactadas y son posibles, a su vez, por la posición intermedia en la que se encuentran estos agentes.

\section{Reflexiones finales}

A partir de este trabajo hemos querido llevar adelante una caracterización de las prácticas de los comisarios, a las cuales no podemos acceder sino es conociendo antes el lugar y el papel de estos agentes en las configuraciones policiales del período. Si bien sobre estas existen estudios finalizados y en curso, los comisarios de ciudad no han sido debidamente atendidos. Sin embargo, a partir de una diversidad de documentación, 
edita e inédita, ha sido posible ubicarlos en su rol de productores de institucionalidad, bien teniendo en cuenta las tensiones, negociaciones e interacciones que esto podía ocasionar. Aunque la extensión y el objetivo de este trabajo no lo permiten, futuros análisis podrán avanzar sobre estos mismos temas en relaciones a otros agentes de la policía, como los alcaldes de barrio.

En el período de transición que nos ocupa no parece extraordinario que, así como la noción de policía es una que se encuentra debatiéndose entre lo antiguo y los nuevos objetivos políticos, en las configuraciones policiales también se producen diversos reacomodamientos que involucran a los comisarios. En parte, quizás por ello sus tareas son tan amplias e incluyen la vigilancia sobre las sociedades negras, sobre el abasto y sobre los corrales de los suburbios de la ciudad. En todas estas funciones, sin embargo, hay un hilo común que está relacionado con el papel de mediación que los comisarios ejercen entre la superioridad policial y el gobierno y otros agentes con funciones policiales, y entre aquellos y los propios vecinos. Esto es, además, en parte facilitado por la inserción de estos comisarios en redes económicas, políticas y familiares conectadas con los grandes procesos de principios del siglo XIX.

La recepción de estas diversas informaciones no es solo trasladada "hacia arriba" o "hacia abajo", sino que es repensada, proyectada y configurada de manera tal que los comisarios imprimen su práctica intelectual en ellas, resultando en la elaboración de modos concretos de controlar y vigilar que, en muchas ocasiones, son aprobados y aceptados por los intendentes y jefes de policía. Resulta de esto que, sin necesidad de forzar una conceptualización de los comisarios como intelectuales, sí es posible abrir el abanico sobre cómo concebimos las funciones y tareas que desarrollaban que, en las fuentes, aparecen como prácticas de tipo intelectual, en tanto no nos encontramos solo con órdenes a seguir, sino con una comunicación bastante fluida en la cual los comisarios participan como productores de disposiciones, reglamentos e informes.

\section{Fuentes}

Inéditas

ARCHIVO GENERAL DE LA NACIÓN (AGN), Sala X, 32.4 .3 (1831-1834-1815).

AGN, Sala X, 32.09.02 (1813).

AGN, Sala X, 32.10.01, Libros 1, 2 y 3. (1812-1822).

AGN, Sala X. 32.10.02. Libros 4 y 5 (1822).

AGN, Sala X, 32.10.05, Libros 12, 13 y 14 (1825-1826). 
ARCHIVO HISTÓRICO DE LA PROVINCIA DE BUENOS AIRES (AHPBA) "Criminal contra Gregorio y su hermano Luis Luxan sobre robos que se le atribuyen por sospecha", Fondo Juzgado del Crimen, C. 34. A. 2. L.34. Exp. 75 (1812).

AHPBA, "Causa criminal contra el autor de la muerte de un negro", Fondo Juzgado del Crimen, C. 34. A.2. L.35. Exp. 11 - (1813).

AHPBA, "Pedro Ramírez por robo", Archivo Histórico de la Provincia de Buenos Aires", Fondo Juzgado del Crimen, C. 34. A. 3. L.44. Exp. 52, (1823).

AHPBA, "Policía pidiendo informe sobre corral del Norte", Fondo Departamento Topográfico, C. 49. A. 2. L. 1. Exp. 7 (1822).

\section{Editas}

ACUERDOS del Extinguido Cabildo de Buenos Aires (1805-1807). Tomo II, Libros LIX a LXII, Serie IV, p. 686 (Buenos Aires, Kraft, 1926).

ACUERDOS del Extinguido Cabildo de Buenos Aires (1810-1811). Tomo IV, libros LXV a LXVII, Serie IV, pp. 118 y 559. (Buenos Aires, Kraft, 1927).

ACUERDOS del Extinguido Cabildo de Buenos Aires (1813-1814). Tomo VI, Libros LXIX a LXXIV, Serie IV, p. 207. (Buenos Aires, Kraft, 1929).

CUTOLO, Vicente (1968). Nuevo Diccionario Biográfico Argentino (1750-1930). Tomos I al VIII. (Buenos Aires, Elche)

ÍNDICE del Archivo del Departamento General de Policía (1859). p. 158 (Buenos Aires, Imprenta de La Tribuna).

JUNTA de Historia y Numismática Americana (1911) La Gaceta de Buenos Aires, reimpresión facsimilar (1810-1821), Tomo III. (Buenos Aires: Compañía Sudamericana de Billetes de Banco).

MANUAL de policía, o leyes y decretos que tienen relación con dicho departamento desde el mes de agosto del año de 1821 (1830), pp. 96-98. (Biblioteca Nacional Argentina (BN), Fondo de la Sala del Tesoro (Tesoro), número topográfico TES3A151321, Imprenta Republicana: Buenos Aires).

MUZZIO, Julio (1920). Diccionario histórico y biográfico de la República Argentina. Tomo I, (Buenos Aires, La Facultad).

PICCIRILLI, Ricardo, ROMAY, Francisco y GIANELLO, Leoncio (1953). Diccionario histórico argentino. Tomos I, II, III, IV, V y VI (Buenos Aires, Ediciones históricas argentinas).

PRADO Y ROJAS, Aurelio (1877). Leyes y decretos promulgados en la provincia de Buenos Aires desde 1810 a 1876, Tomo I y II (Buenos Aires, Imprenta del Mercurio).

QUESADA, Horacio (1942). Papeles del Archivo. (Buenos Aires, Kraft).

REAL ACADEMIA Española (1780). Diccionario de la lengua castellana (Madrid: Joaquín Ibarra. Disponible en: <http://ntlle.rae.es/ntlle/SrvltGUILoginNtlle>. Acceso el 15 mar 2020).

REAL ACADEMIA Española (1817) Diccionario de la lengua castellana por la Real Academia Española. (Quinta Edición. Madrid: Imprenta Real. Disponible en: $<$ http://ntlle.rae.es/ntlle/SrvltGUIMenuNtlle?cmd $=$ Lema\&sec=1.0.0.0.0>. Acceso el: 2 feb 2020).

REGISTRO NACIONAL de las Provincias Unidas del Río de la Plata (1826). Libro Segundo, p. 238. (Buenos Aires: Imprenta de la Independencia)

REGISTRO OFICIAL de la Provincia de Buenos Aires (1879). Pp. 6, 75, 76, 125238 (Buenos Aires, Imprenta de José y Luis Rossi). 
TAU ANZOATEGUI, Víctor (2004). Los Bandos de Buen Gobierno del Río de La Plata, Tucumán y Cuyo. (Época hispánica). (Buenos Aires, Ed. Instituto de Investigaciones de Historia del Derecho).

YABEN, Jacinto (1938). Biografía argentinas y sudamericanas. Tomo I, IV y V. (Buenos Aires: Metrópolis).

\section{Referencias bibliográficas}

ALONSO, Fabián (2001). El delito de la vagancia durante el último cuarto del siglo XVIII. Historias de la Ciudad - Una Revista de Buenos Aires, Buenos Aires, $\mathrm{n}^{\circ}$ 11, pp. 1-6.

ALTAMIRANO, Carlos (2013). Intelectuales: nacimiento y peripecia de un nombre. Nueva Sociedad. Buenos Aires, $\mathrm{n}^{\circ}$ 245, pp. 38-53. Disponible en: <https://nuso.org/media/articles/downloads/3939_1.pdf>

ALZATE ECHEVERRI, Adriana María (2007). Suciedad y orden. Reformas sanitarias borbónicas en la Nueva Granada, 1760-1810. Bogotá: Editorial UAGN.

BARRENECHE, Osvaldo (2001). Dentro de la ley, TODO. La justicia criminal de Buenos Aires en la etapa formativa del sistema penal moderno en Argentina. La Plata: Al Margen.

BARRIERA, Darío (2010). El execrable libro de sus hechos. Cultura jurídica, retórica y deslegitimación de la autoridad en un proceso contra el alcalde del Rosario (18101811). Anuario del Instituto de Historia Argentina. Buenos Aires, $\mathrm{n}^{\circ} 10$, pp. $57-$ 84. Disponible en: http://www.memoria.fahce.unlp.edu.ar/art_revistas/pr.4699/pr.4699.pdf>

BARRIERA, Darío, (2013). Justicias rurales: el oficio de alcalde de la hermandad entre el derecho, la historia y la historiografía (Santa Fe, Gobernación del Río de la Plata, siglos XVII a XIX). Andes. Salta, vol ${ }^{\circ}$ 24, pp. 17-61. Disponible en: <https://www.redalyc.org/articulo.oa?id=12730581001>

BARRIERA, Darío (2017). El alcalde de barrio, de justicia a policía (Río de la Plata, 1770-1830). Nuevo Mundo Mundos Nuevos. Disponible en: <https://journals.openedition.org/nuevomundo/70602>. Acceso el: 7 feb 2020.

BERARDI, Pedro (2018). Territorialidad, profesionalización y política. La construcción de la policía en la provincia de Buenos Aires, 1880-1916. Tesis (Doctorado en Historia). Universidad de San Andrés, Buenos Aires.

BRETAS, Marcos (2011). La policía de la capital del Imperio Brasilero. En: GALEANO, Diego \& Kaminsky, Gregorio (Eds.) Mirada (De) Uniforme. Historia y crítica de la razón policial. Buenos Aires: Teseo, pp. 87-109.

CANDIOTI, Magdalena (2017). Un maldito derecho, leyes jueces y revolución en la Buenos Aires republicana, 1810-1830. Buenos Aires: Didot,

CASAGRANDE, Agustín (2015). Por una historia conceptual de la Seguridad. Los Alcaldes de Barrio de la Ciudad de Buenos Aires (1770-1820). Conceptos históricos. San Martín, $\mathrm{n}^{\circ}$ 1, pp. 40-71. Disponible en: <http://www.unsam.edu.ar/ojs/index.php/conhist/article/viewFile/8/3>

CASAGRANDE, Agustín. (2019) Gobierno de justicia, poder de policía. La construcción oeconómica del orden social en Buenos Aires (1776-1829). Valencia: Tirant.

CILIBERTO, María Valeria (2009). La tierra pública periurbana: Arrendamiento, enfiteusis y ventas en el entorno agrario de Buenos Aires (San José de Flores, 
1800-1862). Trabajos y comunicaciones, La Plata, $\mathrm{n}^{\circ} 35$, pp. 117-147. Disponible en: 〈http://www.memoria.fahce.unlp.edu.ar/art_revistas/pr.4683/pr.4683.pdf>

CILIBERTO, María Valeria y ROSAS PRINCIPI, Andrea (2014). Lo urbano-rural en la historiografía agraria rioplatense. Del final de la Colonia al inicio del siglo XIX. Anuario Colombiano de Historia social y de la cultura. Bogotá, vol. 41, n 2, pp. 261-290.

Disponible en: <https://revistas.unal.edu.co/index.php/achsc/article/view/48788>

DÍAZ DE ZAPPIA, Sandra (2008). La institución del alcalde de barrio en Buenos Aires entre 1810 y 1821: tradición indiana y modificaciones pos revolucionarias. Revista de Historia del Derecho. Buenos Aires, n³6, pp. 45-144. Disponible en: <https://dialnet.unirioja.es/ejemplar/360509>

DUFFAU, Nicolás (2018). La policía en la provincia oriental (1826-1838) Una construcción institucional entre el Antiguo Régimen y el orden republicano. Anuario IEHS. Tandil, vol. 33, pp. 85-105. Disponible en: < http://anuarioiehs.unicen.edu.ar/Files/2018\%201/05\%20Anuario\%20IEHS\%2033 (1)\%20a.Duffau.pdf >

FARGE, Arlette (2008). Efusión y tormento. El relato de los cuerpos. Historia del pueblo en el siglo XVIII. Buenos Aires: Katz.

FAVELUKES, Graciela (2007). 'Para mejor orden y policía de la ciudad'. Reformas borbónicas y gobierno urbano en Buenos Aires. Seminario Crítica IAA. Buenos Aires, $\quad \mathrm{n}^{\circ} \quad 158, \quad$ pp. 1-16. Disponible en: < http://www.iaa.fadu.uba.ar/publicaciones/critica/0158.pdf>

FOUCAULT, Michel (2006). Seguridad, territorio, población. Curso en el Collège de France (1977-1978). Buenos Aires: FCE.

FRADKIN, Raúl (2008). Justicia, policía y sociedad rural. Buenos Aires, 1780-1830. En: BONAUDO, Marta, REGUERA, Andrea y ZEBERIO, Blanca (Coords.) Las escalas de la historia comparada. Tomo 1: Dinámicas sociales, poderes políticos y sistemas jurídicos. Buenos Aires: Miño y Dávila, pp. 247-284.

GALEANO, Diego (2015). O ofício inconfessável: policiais, ordem urbana e mercado de trabalho na cidade de Buenos Aires, 1867-1880. Revista do Arquivo Geral da Cidade do Rio de Janeiro. Río de Janeiro, $\mathrm{n}^{\circ}$ 9, pp. 191-209. Disponible en: < https://www.academia.edu/19535765/O_of\%C3\%ADcio_inconfess\%C3\%A1vel_ policiais_ordem_urbana_e_mercado_de_trabalho_na_cidade_de_Buenos_Aires_1 867-1880>

GALEANO, Diego (2017a). Entre el orden y la fuerza bruta: una historia política de la policía de Buenos Aires, 1852-1880. Ler História, Lisboa, n 70, pp.15-36. Disponible en: < https://doi.org/10.4000/lerhistoria.2705>

GALEANO, Diego (2017b). Genealogía del comisario: policía y orden urbano en Buenos Aires. Iberoamericana. Berlín, vol. 17, n 64, pp. 13-33. Disponible en: < DOI: http://dx.doi.org/10.18441/ibam.17.2017.64.13-33>

GARCÍA DE FLOEL, Maricel (2000). La oposición española a la revolución por la independencia en el Río de la Plata entre 1810 y 1820. Parámetros políticos y jurídicos para la suerte de los españoles europeos. Hamburg: LIT.

GASTELLU, Sofía (2018). La justicia de paz en la ciudad de Buenos Aires. La dimensión territorial de una justicia de proximidad (1821-1854). En: BARRIERA, Darío (Dir.) Justicias situadas. Entre el Virreinato rioplatense y la República Argentina (1776-1864). La Plata: Universidad Nacional de La Plata, pp. 263-290.

GASTELLU, Sofía (2019). Una justicia para el gobierno local: Los jueces de paz de la ciudad de Buenos Aires (1821-1826). Revista de Historia del Derecho. Buenos 
Aires, $\mathrm{n}^{\circ}$ 58, pp. 79-125. Disponible en: <http://inhide.com.ar/portfolio/revista-dehistoria-del-derecho-n-58-julio-diciembre-2019/>

GAYOL, Sandra (1996). Entre lo deseable y lo posible. Perfil de la policía de Buenos Aires en la segunda mitad del siglo XIX. Estudios Sociales. Revista universitaria Semestral. Santa Fe, vol. VI, $\mathrm{n}^{\circ}$ 10, pp. 123-138. Disponible en: < DOI: https://doi.org/10.14409/es.v10i1.2355>

GRAMSCI, Antonio (1999 [1975]. Cuadernos de la cárcel. Edición crítica del Instituto Gramsci a cargo de Valentino Gerratana. Tomo 5. México: Ediciones Era.

HERRERO, Fabián (2018). Voces de invasión e iniciativas disruptivas en Buenos Aires en los primeros años de la década de 1820". Claves. Revista de Historia. Montevideo, vol. 4, $\mathrm{n}^{\circ}$ 6, pp. 95-124. Disponible en: <DOI: https://doi.org/10.25032/crh.v4i6.184>

HERRERO, Fabián (2007). Movimientos de Pueblo. La política en Buenos Aires luego de 1810. Buenos Aires: Ediciones Cooperativas.

INFESTA, María Elena (2006). La pampa criolla. Usufructo y apropiación privada de tierras públicas en Buenos Ares, 1820- 1850. Mar del Plata: Eudem.

IRAOLAGOITIA, Gonzalo (2014). Territorio, autoridad y Estado: comisarías de distrito y juzgados de paz en la campaña sur santafesina, 1854-1883. Tesis (Maestría en Historia), Universidad de San Andrés, Buenos Aires.

KALIFA, Dominique y KARINA-COHEN, Pierre (2008). Le commissaire de police au XIXe siecle. París: Publications de la Sorbonne.

KAPLAN, Steve (1981). Notes sur les commissaires de pólice de Paris au XVIIIe siecle. Revue d'histoire moderne et contemporaine. París, vol 28, $\mathrm{n}^{\circ} 4$, pp, 669686. Disponible en: < https://www.persee.fr/doc/rhmc_00488003_1981_num_28_4_1171>

LANTERI, Ana Laura (2015). Se hace camino al andar. Dirigencia e instituciones nacionales en la "Confederación" (Argentina, 1852-1862). Rosario: Prohistoria.

LEVAGGI, Abelardo (1976). La seguridad en la campaña bonaerense entre los años 1821 y 1826. Establecimiento, supresión y restablecimiento de las comisarías de policía de campaña. Investigaciones y ensayos. Buenos Aires, $\mathrm{n}^{\circ}$ 20, pp. 377-410.

LEVAGGI, Abelardo (2009). La alcaldía de hermandad en el virreinato del Río de la Plata (1776-1810) (Casuística y jurisprudencia). Revista de Estudios HistóricoJurídicos. Valparaíso, $\mathrm{n}^{\circ}$ 31, pp. 317-348. Disponible en: $<$ https://scielo.conicyt.cl/scielo.php?script=sci_arttext\&pid=S071654552009000100012>

MADRID, Rufino Manuel (2007). Vencer la noche. La Sevilla iluminada (historia del alumbrado público de Sevilla). Salamanca: Kadmos

MIRANDA OJEDA, Pedro (2013). Las comisarías inquisitoriales en la provincia de Yucatán (SS. XVI-XIX). Astrolabio. Córdoba, ${ }^{\circ}$ 11, pp. 43-59. Disponible en: <https://revistas.unc.edu.ar/index.php/astrolabio/article/view/6306>

MOLINA, Eugenia (2010) Los funcionarios subalternos de justicia en Mendoza, 18201852: entre el control comunitario y el disciplinamiento social. Nuevos mundos, mundos nuevos. Disponible en <https://journals.openedition.org/nuevomundo/59353>. Acceso el: 18 mar 2020.

PIAZZI, Carolina y RÍOS, Evangelina (2012). Comisarios de campaña en el departamento Rosario: entre ocupaciones públicas e intereses privados (18501865). En: GARAVAGLIA, Juan Carlos, PRO RUIZ, Juan y ZIMMERMANN, Eduardo (Eds.) Las fuerzas de la guerra en la construcción del Estado: América Latina, siglo XIX. Rosario: Prohistoria, pp. 381-412. 
PIMENTA, Joao Paulo (2013). Las independencias cruzadas de Brasil e Hispanoamérica: el problema de las sincronías y diacronías. En: THIBAUD, Clement, ENTIN, Gabriel y GÓMEZ, Alejandro y MORELLI, Federica (Dirs.). L'Atlantique révoluctionaire. Une perspective ibéroaméricaine. Berechel: Ed. Les Perséides, pp. 289- 299.

PROCHASSON, Christophe (2003). Sobre el concepto de intelectual. Historia Contemporánea. Lejona, $\mathrm{n}^{\circ}$ 27, pp. 799-811. Disponible en: < https://www.ehu.eus/ojs/index.php/HC/article/view/5217/0?source=/ojs/index.php /HC/article/view/5217/0>

RICO, Alejandra (2007). Jueces Comisarios. La justicia de paz y las funciones policiales en la provincia de Buenos Aires durante el período de Rosas. En: XI Jornadas Interescuelas / Departamentos de Historia. San Miguel de Tucumán: Departamento de Hiustpria. Facultad de Filosofía y Letras de la Universidad de Tucumán, pp. 1-21. Disponible en: <https://www.aacademica.org/000-108/928>. Acceso el: 17 mar 2020.

SCHLEZ, Mariano (2010). El bando de los opresores. La derrota política y social contrarrevolucionaria en Buenos Aires (1810-1816). Historia Caribe. Barranquilla, vol. $\mathrm{V}, \quad \mathrm{n}^{\circ} 16$, pp. 105-125. Disponible en: < https://dialnet.unirioja.es/servlet/articulo?codigo=3644156>

SCHLEZ, Mariano (2013). Los circuitos comerciales tardo-coloniales. El caso de un comerciante monopolista: Diego de Agüero (1770-1820). Tesis (Doctorado en Historia). Universidad de Buenos Aires, Buenos Aires.

SZUCHMAN, Mark (2001). Constructing the city, constructing the state. Arquitecture and political transition in urban Argentina, 1810-1860. En: Uribe Uran, Víctor (Ed.) State and society in Spanish America during the age of revolution. Delaware: SR Books, pp. 193-218.

TERNAVASIO, Marcela (2004). Construir poder y dividir poderes. Buenos Aires durante la 'feliz experiencia' rivadaviana. Boletín del Instituto de Historia Argentina y Americana Dr. Emilio Ravignani. Buenos Aires, $\mathrm{n}^{\circ} 26,3^{\circ}$ serie, pp. 7-43. Disponible en: < http://repositorio.filo.uba.ar/handle/filodigital/7092>

VACCARONI, María Agustina (2014). Las Órdenes Superiores: Gobierno, policía y sectores subalternos. Revista Electrónica de Fuentes y Archivos del Centro de Estudios Históricos Prof. Carlos S. A. Segreti, Córdoba, no 5, pp. 142-151. Disponible en: < https://refa.org.ar/file.php?tipo=Contenido\&id=215>

YANGILEVICH, Melina (2018). Prefecturas, comisarías de campaña y construcción estatal en la Provincia de Buenos Aires (Argentina) a mediados del siglo XIX". Secuencia. México DF, $\mathrm{N}^{\circ}$ 102, pp. 70-99. Disponible en: < http://secuencia.mora.edu.mx/index.php/Secuencia/article/view/1359>

Artigo recebido em 20 de março de 2020

Aprovado em 11 de junho de 2020

DOI: 10.12957/intellectus.2020.49424 